\title{
Complex Metadata management in Earth Observation for environmental research
}

\author{
M. Zingler, H. Pintaritsch \\ European Space Agency - ESRIN \\ Via Galileo Galilei, I-00044 Frascati, Italy, Tel. +39.6.94180.1, \\ mazi@mail.esrin.esa.it
}

\begin{abstract}
User expectations and requirements for access to Earth Observation data and information are constantly increasing. Recent improvements in open system architectures and networks have intensified discussions on interoperability and data availability. Efficient Metadata management software supports the search for data and the final retrieval from EO archives. The European Space Agency is challenged to provide solutions for that objective. Environmental research applications will benefit from enhanced metadata layer functionality to access EO products.
\end{abstract}

\section{Keywords}

EO metadata, inventory system, provider/subscriber concept, thematic access

\section{INTRODUCTION}

Earth Observation (EO) is the combined effort of technical and scientific resources to achieve a variety of goals e.g. understanding of large scale phenomena on earth (Global Change), generation of maps and recording of atmospheric processes for meteorology. A large part of the EO user services manages metadata (MD) with the objective to lead users to the products they need in their application domain. MD should present a summery on each required data set. MD systems can assist the user during the process of mining into data collections he is interested in. The MD models might also be exchanged between systems for interoperability purposes.

The European Space Agency takes a major share in the EO framework through its remote sensing satellites and the operations of ground segment $(\mathrm{G} / \mathrm{S})$ facilities. The EO G/S should foster the completeness, integrity and accessibility of the data obtained from the instruments. 
The capability of accessing even small portions of data with short response times has to be ensured in spite of a rapidly increasing volume of stored data (up to Tera- or Pentabytes).

The proposed data management principles discussed below focus on operations on MD as required by satellite data inventory systems.

\section{THE EARTH OBSERVATION USER SERVICES FRAMEWORK}

Earth Observation covers large geographical areas, but records as well small scale processes and features. This is a result of the increasing precision and resolution of the instruments. By its nature EO crosses boundaries and thereby becomes an international issue. EO has to consider the interests of local groups with a large geographical distribution. The broad variety of scientific and technical applications with their demand to perform cross-disciplinary data mining (Thieman 1992) is relevant to EO system design.

The requirements arising from the mission to serve those communities pose complex architectural design tasks on the agencies. Our target user communities are located in the scientific community (research institutes, universities, etc.), institutional community (meteorological offices, governmental institutions, etc.) and the commercial community (value-adding services, cartographic services, environmental services, etc.). The European Space Agency undertakes efforts in collaboration with the EC to create an European Earth Observation System that will cope with user group and system heterogeneity. NASA's EOSDIS can be named as such a system, although being adjusted to the scientific community (Elkington 1995).

Complex requirements stem originally from the scientific community. Now commercial interests play more and more a substantial role in the advertisement and distribution of EO data, and some industrial activities depend on high quality data with high reliability in distribution (e.g. regular Fast Delivery Products for ship route planning). Figure 1 shows the modular character of the overall ESA EO data and user services capable of:

- Advertising data acquisition possibilities,

- Advertising products, services and costs,

- Providing user tools and system access gateways,

- Providing service navigational tools, Directories, Guides and tutorials,

- Providing inventory and browsing facilities (including CD-Browsers),

- Providing Data Dissemination facilities,

- Providing user registration, access control and billing,

- Providing Subscription services of functions described above.

An EO data and service site assembles several of the above components. They have to cooperate at and between distributed sites, which requires interoperability layers that act as gateways from/to client systems to the various server systems. Depending on the type of services the interoperability layer will be achieved through concepts from common protocol for information request and exchange up to direct access to resources at the sites. The user system domain is coupled via firewalls to a dedicated operations system domain and servers. A complex cooperative hypertext Guide and Directory systems forms part of the advertiser and navigation role of the system. 


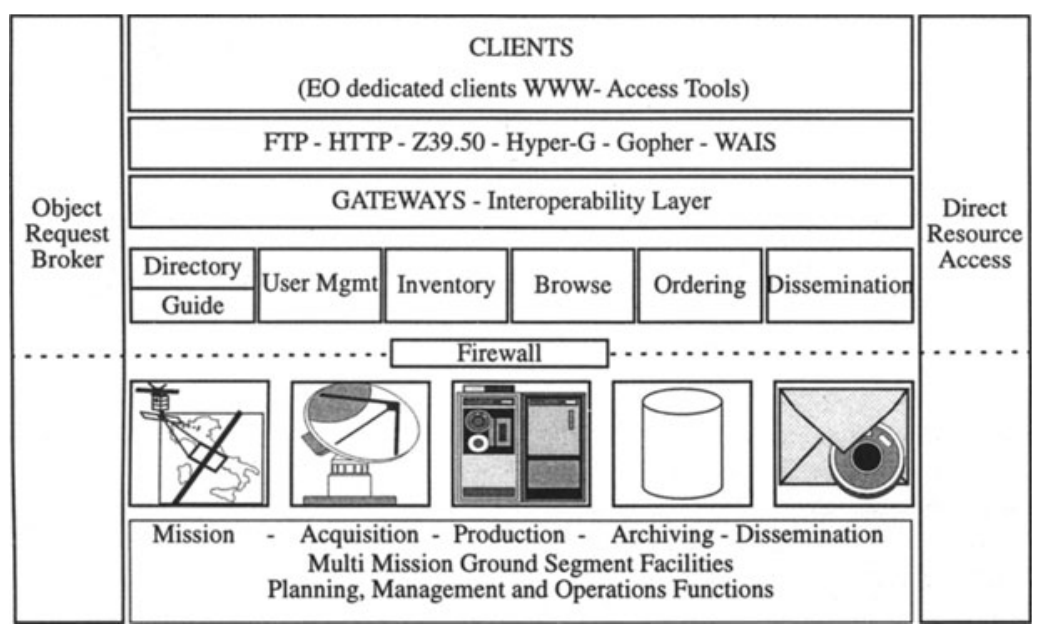

Figure 1: EO User Services Conceptual Model

The system design has to anticipate a constant growth of data, notably when automated classification of EO products becomes more common and adds attributes and relationships to the model (Cromp et al., 1992). An adequate data architecture in the EO system (Childs 1991) and a consistent modelling of EO (meta-)data are the basis for high-level interoperability. As an additional aspect, user server systems have to provide flexible service facilities (e.g WWW, Object Broking), while the operational side requires robustness and stability. The latter has to maintain the availability and integrity of the satellite data for 15 to 50 years.

\section{A METADATA CONCEPT IN EARTH OBSERVATION}

A focal point for users of EO information is the knowledge about existing data sets (collection of granules) including related auxiliary data as obtained e.g. from ground campaigns. The granules from that data are stored in distributed archives, where only partial coherency of granules is achieved since the conditions of data reception and post-processing are varying from center to center. Granules are the smallest units of an identifiable data sequence, e.g an image.

Metadata describing each granule, called Metagranule (MG), can be created at the planning stage of a granule (for future satellite acquisition) or upon registration. Their lifecycle is determined by the status of the granules they relate to. The MG makes particular sense through the object-oriented modelling by which the rigid record and table driven model is broken up and the underlying granule is described more accurately.

In general, the metadata (MD) describe the physical and logical properties of the stored data sets and consist of textual information as well as low resolution browse images or statistical summaries of granule properties. The attributes in a metagranule are determined by various sources (user, telemetry, production etc.). MGs might be aggregated up to an acceptable temporal granularity (Hood et al. 1991). MD allow users to judge if data sets are suitable for their 
application and form an important element in the chain from user's first contact with an EO advertising service to his final order of a product. Browse products (low resolution products) and directory information (i.e. a hierarchical representation of available products) can also be regarded as metadata and will be logically co-located and accessible with the textual inventory information for a product.

A data pyramid reflects the data model that must be supported with each level having an index to the lower level in the pyramid, showing the increasing accessibility to the top and the large volume of products at the bottom of the pyramid. The low volume of condensed directory information is the located at the top. We extended the pyramid representation as proposed by (EOSDIS 1994) to a data rhombus to show the related service function and its properties with low $(0)$ and high $(\infty)$ requirements. The embodied data mining process is the summery of the user's activities to obtain the target product and is discussed later.
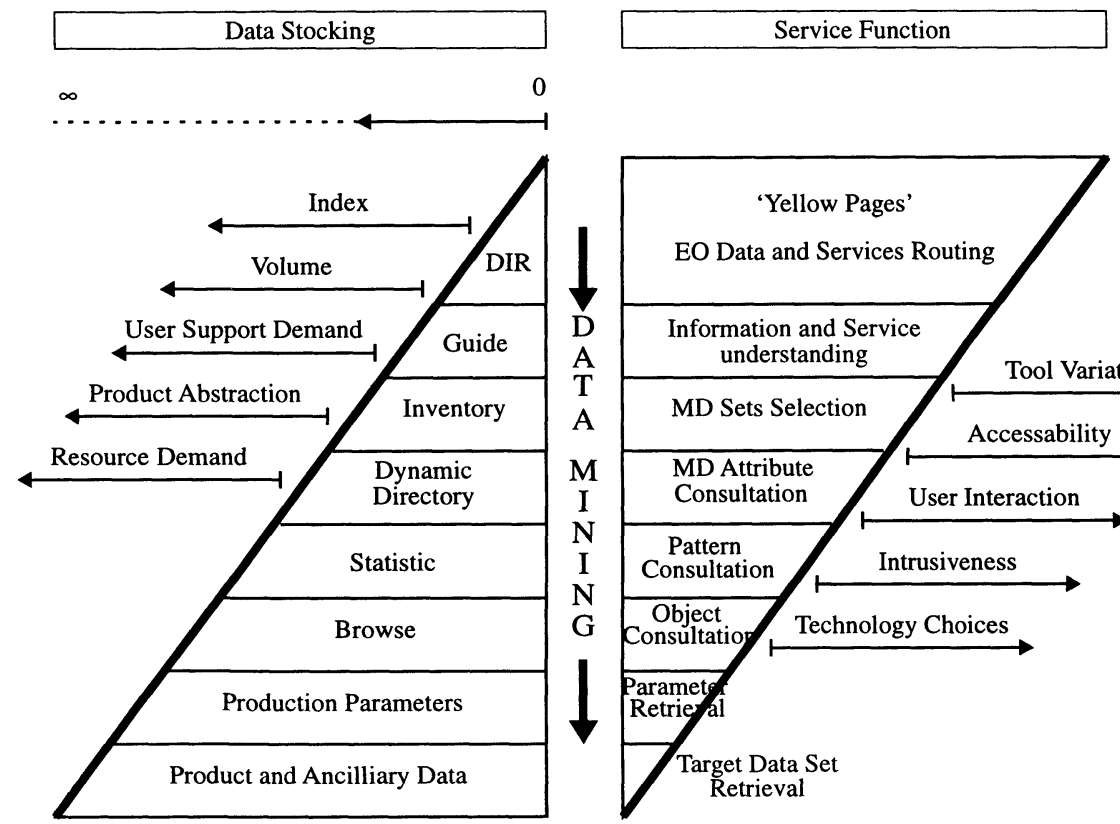

\section{0}
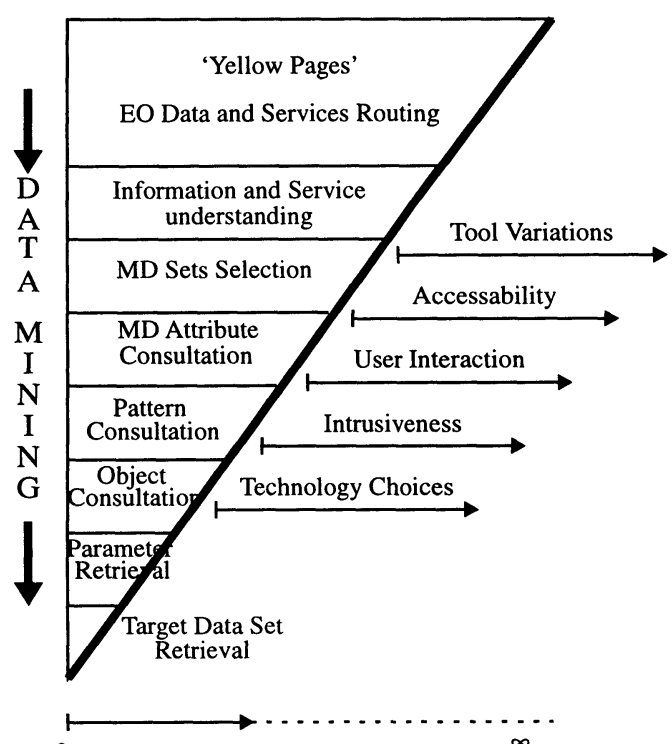

0

Figure 2: Data and Service Function Rhombus

The distribution of providers and users and the dispersed service nodes enforce global and local metadata models. The system analysis process had to find mechanisms to ensure that the system could cope with the parameter variation in the model. A major role plays the possibility to assign to each MG type particular interpretation, processing and referencing methods as they are required by each user group. These are best implemented as methods on object data. MMIS (see below) provides an integrated schema evolution mechanism for the complex MD 
structure. It is written in an IDL-like fashion and called Universal Inventory Definition Language (UIDL). User views, views by the updating entities and the internal OODBMS schemas are defined in a single description process and are compiled into an Interface Definition Object. This can be communicated directly to other federated inventories of the same kernel type. It supports therefore ideally the Provider/Subscriber concept and could contribute to the implementation of a more global information resource dictionary as described in (Hsu et al., 1991).

We consider the following five mechanisms as relevant to metadata management:

- the provider-subscriber concept for a controlled data replication to distributed systems.

- the thematic access to improve access and performance in inventories.

- the object oriented modelling.

- data mining support.

- the UIDL to communicate the three-folded data structure.

\section{METADATA MANAGEMENT IN THE MULTI MISSION INVENTORY SERVICE SYSTEM (MMIS)}

Increasingly, the object-oriented paradigm is applied in payload data systems design, metadata modelling and management. Major constituents in related applications are object-oriented modelling techniques, Object-Oriented Database Management Systems and Object-Request Broker technologies. The Multi Mission Inventory Service System (MMIS, Figure 3) is a development managed by ESA/ESRIN demonstrating new concepts of metadata management based on the above technologies. It is one system component of the Multi Mission User Information Services (MUIS) which is based on a conceptual architecture as shown in Figure 1.

Metadata, generated or available at distributed sites, is kept in the inventory which is established as an object-oriented distributed database (Öszu 1991). The main EO inventory application qualities are:

- The DB application is permanently loaded by a stream of updates from the various sources (high operational transactions). The share of Near-Real-Time updates will be a factor for the future (NRT product availability and management).

- The actual user transactions (queries) load is still low, but will dramatically rise when the system is on-line over the WWW on Internet (expected are 500 to 10.000 queries daily).

- The distributed function across the provider/subscriber links will impact performance requirements, since the replication mechanism must access consolidated MD in the provider DB.

The task in the federated inventory network is to allow access to global and local metadata schemas and to pass context information in case of distributed searches and service requests during user sessions to other sources of information. An object and service broker will dispatch requests and returned information. The interoperability on the advertiser level will be achieved over HTTP with some extensions to allow the invocation of sophisticated server functionality which then uses e.g. DCE or CORBA.

The logical MD management architecture is a mixture of a cooperative C/S (Client/Server) architecture and federated DB systems. The C/S cooperation results from the way how end- 


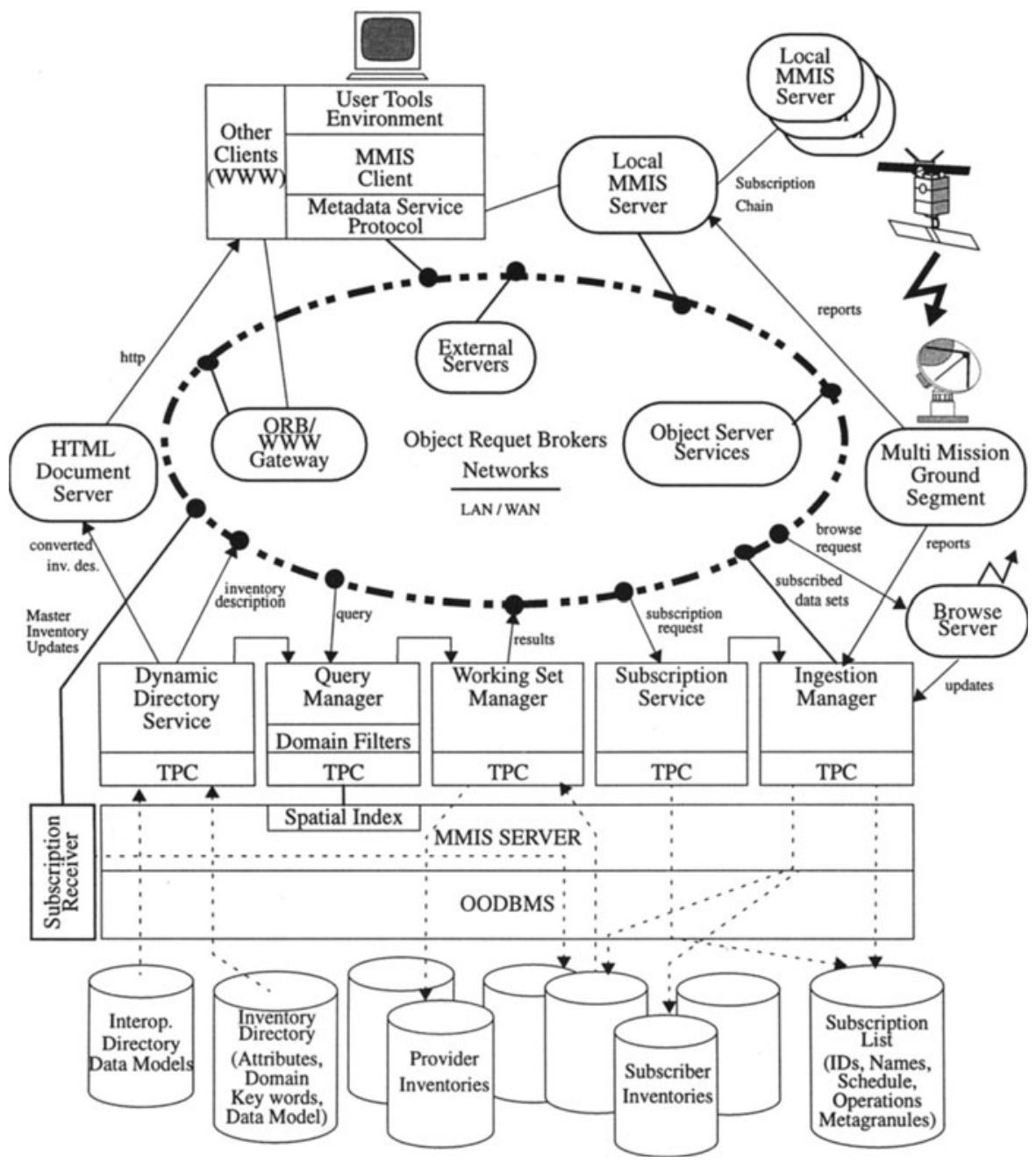

Figure 3: MMIS Services and Conceptual Architecture

user tools interact locally and over large geographical distances. Another factor is the user's heterogeneous environments with discrepancies in connectivity and performance. The MMIS protocol considers restrictions on the client side as it allows server oriented operations and pulling of results in chunks from the client working set. The main concepts for distribution are the: 
- Federated system components which work on a common 'global schema'.

- Cooperative system components that announce their local schema through a Dynamic Directory which can be queried just like the main inventories. The Dynamic Directory can be converted on demand into documents to allow text searching for other interface types particularly those which apply a knowledge based semantic system support.

The metadata management systems have to survey the flow of metadata according to a highlevel strategical operations plan (system initiators driven) and a management operations plan which are both driven by the system characteristics and the short term objectives.

The metadata management must cope with the complexity of the management rules including fault tolerance and should guarantee the consistency of the information between a central site and the distributed sites (Zingler 1995).

\section{PROVIDER/SUBSCRIBER CONCEPT FOR A DISTRIBUTED METADATA MANAGEMENT}

The Provider/Subscriber (P/S) concept is based on the notion that other sites want to or must receive a set of metadata on a regular bases. The subscription is important since it allows to establish a persistent logical link between the subscriber and the provider. This principle, implemented in the inventory system software, supports the metadata management in an architecture of centralized and decentralized EO services.

Users can subscribe to up-to-date information on products they need. Operations as replicate, update and delete on the data sets determine the level of data consistency amongst the distributed copies depending on the moment they are initiated compared to the master inventory update. A P/S protocol had to be built in MMIS which maintains a list of subscribers and operations that are requested by those subscribers. The mechanism is based on the ORB with a scheduler and can enforce operations also after network link interruptions. The subscription can be chained to give remote sites the possibility to act in turn also as a provider. We have considered this approach as fitting the operational needs of inventory servers. Nevertheless it can be complemented later by a client driven status update as described in (Joy et al. 1992).

\section{MODELLING OF EO METADATA}

The modelling of EO metadata has to cope with a variety of instrument and data types and their properties in terms of:

- Frequencies and techniques (spectral ranges, multi-channel devices, active, passive),

- Coverage (temporal and spatial scale, orbit characteristic, repetition or permanent coverage, instrument footprint),

- Resolution (spatial, temporal, radiometric, spectral resolution),

- Instrument geometry (nadir- and limb-looking, side-looking, view-angles),

- Calibration (information about stability, device and data calibration),

- Data rates and observation of events (e.g. volcano eruption process). 
There is large amount of different instruments for different purposes. The main classes are Spectrometers (spectral), Radiometers and Scatterometers (intensity), Imagers, Sounders and Altimeters (spatial) (Kramer 1992). Hence, dozens of product parameters exist and the variation in parameter characteristics is so large, that its impossible to take them into account a-priori a system design (Hachem et al. 1990). Especially the geometry (footprints) of the instruments is noteworthy, since it is special challenge to databases that have to keep a knowledge of the spatial reference of each product.

The management of EO data is confronted with the challenge to handle complex data structures. Products of the most advanced instruments contain in addition to temporal, spatial and instrument specific information, high level quality and processing parameters. Experienced users want to create queries that include a combination of those parameters, and sometimes even post-processing algorithms on them (e.g. restricted view angle of instruments).

This aspect leads to the creation of a data model and database indices which are based on previous experiences of average user behavior. Nevertheless they have to be flexible enough to be extended when new user communities set up new priorities.

The high importance of spatial information in metagranules led to the use of $\mathrm{R} *$ trees as the basic indexing method (Guttman 1984, Beckmann et al. 1990). The nodes in the trees are minimum bounding rectangles (MBRs) of arbitrary shapes, e.g. polygons. This indexing technique suits the use of virtual metagranules which can consist of references to several real metagranules. The combined information is transparent to the user, who can search on them just as on usual data.

\section{DATA MINING AND THEMATIC ACCESS TO EO METADATA}

The data access activities of users are characterized by an interest in a specific application area that he or she is working in. It is important to the acceptance of the system by the user that required information is available at the right time. Hence, the system analysis process has to consider 1) the EO User Access Model, defined by specific logical operators and reflecting typical user behaviour, and 2) the EO System Metadata Model, describing data structures and properties and stating the physical availability of metadata that is distributed over different repository sites.

The data mining problem described here comprises the search from top-level interactive (searchable) guides and directories, inventories, browsers and order services to a low-level archive oriented product query and/or retrieval mechanisms. Those queries and commands can be issued by the user and also by the system itself. An 'advertise' service, available at the level of the guide subsystem, supports the entry to the system and is linked to the metadata. The further data mining process follows the logic in Figure 2, the data rhombus.

A solution to multi-discipline query processing is to provide a metadata retrieval mechanism that considers the variety of thematic query combinations. The search engine has to recognize that the query matches a thematic pattern and traverses the index tree. It is made of two layers:

- the thematic layer organizing application typical queries into an index structure.

- the spatial and temporal layer reflecting the common reference properties of MGs. 


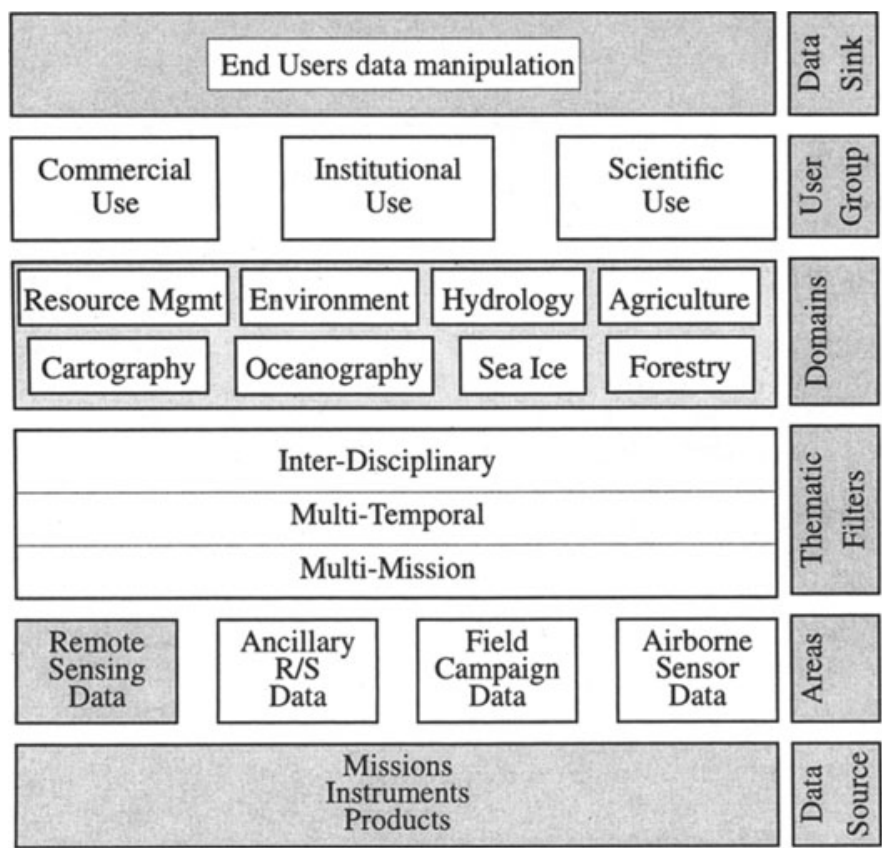

Figure 4: EO data layers

This mechanism known as thematic access is a fundamental approach for efficient searches in large scientific metadata repositories (See Figure 4). It can replace or complement ad hoc object-oriented query languages. Another important aspect is the availability of metadata to users. This is achieved either simply by conveniently putting metadata onto a local server, or also by the temporal requirements of certain application for regular updates. The availability of metadata is ensured from the user perspective by:

- subscription to a particular set of metagranules specifying the thematic and other properties supplied by a provider,

- directory services announcing hierarchically the existence of data sets (yellow pages).

In the MMIS data is arranged and partitioned in advance, creating domains. This significantly increases the precision of search results and reduces the response time. Domains will naturally be created for spatial and temporal criteria, and lead therefore to the common use of abbreviated search parameters (e.g. the user can directly search for the region 'Italy' instead of approximating its borders with a polygon). This differs from the approach of synonym lists.

As OODBMS allow several entry points into a database, every domain can immediately be accessed. Additionally, the partitioning of domains can be done recursively, making domains composed of several subdomains. This method is used to improve the database 'layout' and significantly reduces search times. 
In the $\mathrm{OO}$ environment the domain concept can very easily be applied to all metagranule parameters, i.e. grouping can be done on qualification parameters. Using this technique, data from different satellite missions but with similar parameters can e.g. be grouped to form a domain of 'Oceanography' to see the effects of environmental change over several years, seen by various instruments and satellites

\section{FUTURE IMPROVEMENTS TO EO METADATA SERVICES}

The move from a canonical query form to real object-oriented queries is a step that is induced where metadata with a complex model has to be searched and also where location transparency should be achieved through the Object Request Broker mechanism. There are practical considerations that derive first an object oriented ad hoc query language (OQL) whereas the $O O$ query object approach can increase the performance and reliability in a distributed system.

The system evolution has to provide search and management mechanisms that guarantee in the future a low-level access directly to archived granules, using the index system mentioned above with metagranules pointing directly to the archive. Since the complexity of that search can be considerably high an invocation of search-agents launched at the user discretion is proposed. The agent would interface with the storage management system, complete a search matching the criteria and react on temporal constraints at the host system. The agent-driven approach search increases the abstraction of data location and allows to return different data representations besides other functionality that can be off-loaded from the local user client.

An important step forward in the area thematic access will be the integration of a knowledgebased methods that optimize and expand the thematic index substructure of the inventory.

\section{CONCLUSION}

The management services of actual and future EO systems follow generally the tradition of distributed information systems, but add more complexity to it through the implementation of a highly flexible distribution. This is and will be enforced by local, regional and global data location and distribution requirements. The metadata handling is an important element in the EO system design because it describes what kind of data sets the system preserves in its storage. The user must able to reach through the metadata level the required data set.

The solutions found for the management of EO metadata in a highly distributed environment and the design of ESA/ESRIN's Multi Mission User Information Services must provide the needed facilities. As demonstrated before, some solutions should be extensible to other similar scientific and environmental applications.

\section{REFERENCES}

Beckmann N. et al. (1990) The R*tree: An Efficient and Robust Access Method for Points and Rectangles. Proc. of ACM SIGMOD Int. Conference on Management of Data, 322 - 331.

Childs D.B., (1991) Data Architecture and the Data Architect, in Earth and Space Science Information Systems (ed. A. Zygielbaum), Conf. Proc., 50-564. 
Cromp R.F., Campbell W.J., Short N.M. (1992) An Intelligent Information Fusion System for Handling the Archiving and querying of terabyte-sized spatial Databases, in Earth and Space Science Information Systems (ed. A. Zygielbaum), Conf. Proc., 586-597.

Elkington M.D. (1995) EEOS Concept Study - EOSDIS Architecture Review, ESA/ESRIN Contract P/WS/KH/9681.

EOSDIS (1994) System Design Specification for the ECS Project, Document Number: 207CD-001-001, 6/94.

Guttman A. (1984) R-trees: a dynamic index structure for spatial searching. Proc. of ACM SIGMOD Int. Conference on Management of Data, 47 - 57.

Hachem N.I., Gennert M.A., Ward, M.O. (1990) A DBMS Architecture for Global Change Research, in Earth and Space Science Information Systems (ed. A. Zygielbaum), Conf. Proc., 121-131

Hood C.A., Howie R., Verhanovitz R. (1991) The development of a Prototype Level-Three Interoperable Catalogue System, in Earth and Space Science Information Systems (ed. A. Zygielbaum), Conf. Proc, 152-165

Hsu C. (1991) Information Resources Management in Heterogeneous, Distributed Environments: A Metadata Approach, IEEE Transact. on S/W Eng., Vol. 17, No. 6, 6/91.

Joy S.P., King T.A., Walker R.J. (1992) Automating Database Management for Distributed Database Systems, in Earth and Space Science Information Systems (ed. A. Zygielbaum), Conf. Proc, 444-453.

Kramer H. J. (1992) Earth Observation Remote Sensing - Survey of missions and sensors, Springer Verlag, $200 \mathrm{ff}$.

Özsu M.T., Valduriez P. (1991) Principles of Distributed Database Systems, Prentice-Hall, 66 ff., $494 \mathrm{ff}$.

Thieman J.R. (1992) Identifying, Accessing, and Interoperability using Earth and Space Science Information Systems, in Earth and Space Science Information Systems (ed. A. Zygielbaum), Conf. Proc., 871 - 883

Zingler, M. (1995) Multi Mission Inventory System Development Guideline, Technical Note, ESA/ESRIN, MMIS-DGD-TN/2.0

\section{BIOGRAPHY}

Matthias Zingler received a MSc degree as an engineer for Aerospace and Space Technology from the University of the Armed Forces, Munich, 1986. He worked in the area of missile airdefence systems and joined the Nato Programming Center, Belgium, in 1989 for developments in missile command and control system software. Since 1993, being with the European Space Agency as a Technical Officer in Italy, he manages component design and development in satellite payload data ground systems.

Hannes Pintaritsch received a MSc as an engineer for applied mathematics and informatics at Graz University of Technology, Austria, 1993. His thesis was partly funded by Hewlett-Packard as part of a 2 years information retrieval project. He joined the European Space Agency in 1994 as young graduate software engineer for object-oriented systems and databases. 